\title{
Dexterity augmentation on a synergistic hand: the Pisa/IIT SoftHand+
}

Cosimo Della Santina, Giorgio Grioli, Manuel Catalano, Alberto Brando, Antonio Bicchi

This is the accepted version of an article published in 15th IEEE-RAS

INTERNATIONAL CONFERENCE ON HUMANOID ROBOTS, pp. 497-503

DOI: 10.1109/HUMANOIDS.2015.7363595

(C) 2015 IEEE. Personal use of this material is permitted. Permission from IEEE must be obtained for all other uses, in any current or future media, including reprinting/republishing this material for advertising or promotional purposes, creating new collective works, for resale or redistribution to servers or lists, or reuse of any copyrighted component of this work in other works. 


\title{
Dexterity augmentation on a synergistic hand: the Pisa/IIT SoftHand+
}

\author{
Cosimo Della Santina ${ }^{1}$, Giorgio Grioli ${ }^{2}$, Manuel Catalano ${ }^{2}$, Alberto Brando ${ }^{2}$ and Antonio Bicchi ${ }^{1,2}$
}

\begin{abstract}
Soft robotics and under-actuation were recently demonstrated as good approaches for the implementation of humanoid robotic hands. Nevertheless, it is often difficult to increase the number of degrees of actuation of heavily under-actuated hands without compromising their intrinsic simplicity. In this paper we analyze the Pisa/IIT SoftHand and its underlying logic of adaptive synergies, and propose a method to double its number of degree of actuation, with a very reduced impact on its mechanical complexity. This new design paradigm is based on constructive exploitation of friction phenomena. Based on this method, a novel prototype of under-actuated robot hand with two degrees of actuation is proposed, named Pisa/IIT SoftHand+. A preliminary validation of the prototype follows, based on grasping and manipulation examples of some objects.
\end{abstract}

\section{INTRODUCTION}

Still nowadays, the design and realization of dexterous robotic hand keeps being a big challenge in the field of robotics. Over the years, several hand design were proposed to try and match the level of dexterity of the human hand. These hands typically resort to very complex and articulate designs which cleverly integrate many actuators, sensors and joints trying to get close to the richness and complexity of the sensory and motor functions of the human hand which, nevertheless, still remains a goal on the horizon (e.g. [1]).

An alternative and promising trend in robot hand design is simplification to trying encompassing some of the limitations of overly complex mechanical system, the removal of some of its components can introduce more advantages than drawbacks, if done with the right criteria. One of the best simplification criteria is that of embedding part of the control intelligence in the physical structure of the system itself, the main tool to achieve this goal is under-actuation [2]. Thanks to under-actuation designers can reduce the number of degrees of actuation (DOAs) of robotic hands and thus simplify their design. While in a traditional robot hand a reduction of DoAs would imply less DOFs and, in turn, a considerable reduction of shape adaptation capabilities, under-actuation, gives principles to design hands that retain a large number of DOFs and, by consequence, adaptability. To implement this, under-actuation resorts to differential transmissions, realized in various forms, e.g. gears [3], tendon and pulleys [4].

This work is supported by the European Commission Grant no. H2020ICT-645599 "SOMA": SOft MAnipulation and the ERC Advanced Grant no. 291166 "SoftHands"

${ }^{1}$ Research Center "Enrico Piaggio", University of Pisa, Largo Lucio Lazzarino 1, 56126 Pisa, Italy

${ }^{2}$ Department of Advanced Robotics, Istituto Italiano di Tecnologia, via Morego, 30, 16163 Genova, Italy

cosimodellasantina@gmail.com

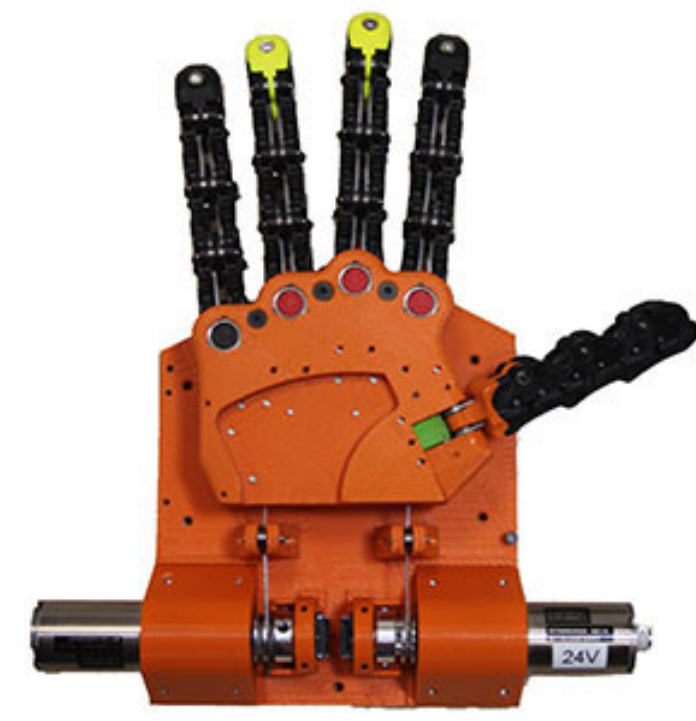

Fig. 1. Prototype of the Pisa/IIT SoftHand+, an evolution of the Pisa/IIT SoftHand with an additional DOA, powered by a friction based transmission.

Another idea, coming from motor control [5], recently reached a great interest in the robotic hands field: postural synergies. Synergies can be defined [6] as a set of variables that show correlated changes in time or with changes in task parameters. Postural synergies can be seen as a sort of basis of the subspace of the effective human movement among all those made possible by the kinematics of the body. Moreover, synergies can be ordered in terms of the statistical variance of the total amount of motion that they explain. This makes them a successful tool for artificial hand analysis, control and design simplification [7]. Notorious examples are [8] and [9].

The basic concept of postural synergies later evolved both on the human motor control and robotic sides. In particular the Soft synergies theory sprouted up [7], which assumes that synergies exist on an ideal domain, and define a virtual reference movement, toward which the physical system is elastically attracted. The manipulated environment, in turn, opposes to the ideal hand motion through its own compliance. The two actions concur to the generation of an equilibrium (e.g. [10] [11]). Based upon the theory of soft synergies, adaptive synergies [12] integrated synergies with under-actuation, yielding a simple implementation of the former, with a series of considerable advantages, such as control and design simplification.

The Pisa/IIT SoftHand [13] is the most recent outcome of this research (see figure 3(a)): it implements one soft synergy, 


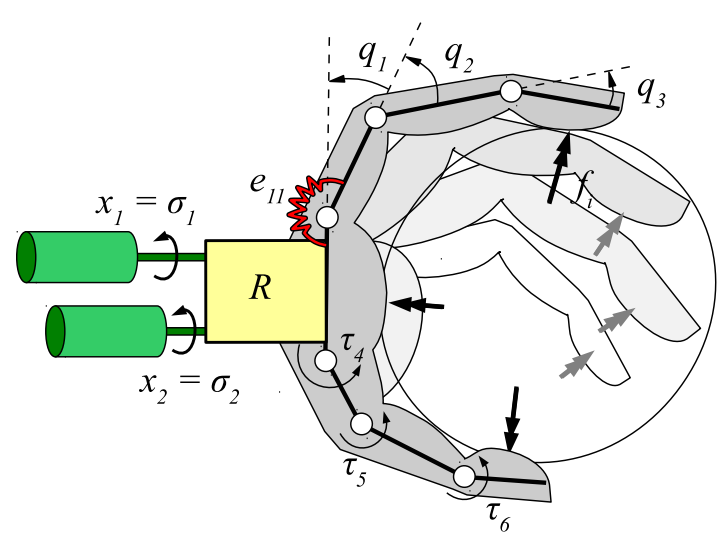

Fig. 2. Schematic of a robotic hand with adaptive synergies grasping an object. The prime movers, on the left (in green) generate motion acting on the angles $\sigma$. Those motions are mapped to the hand joint angles $q$ through the matrix $R$, which collects the transmission ratios (inside the yellow box). The final hand posture depends on the external wrenches $f_{\text {ext }}=\left[f_{1}^{T}, f_{2}^{T}, \ldots\right]^{T}$, the internal torques $\tau_{a}=$ $\left[\tau_{1}, \tau_{2}, \ldots\right]^{T}$ (due to actuation) and the (red) springs elasticity (matrix $E$ collects all the stiffness in its elements $e_{i, j}$ ).

actuated with a transmission system that uses one tendon, pulleys, and one motor. The SoftHand demonstrated excellent grasping skills, in many different situations, combined with robustness and a simple control interface ${ }^{1}$.

Having reached such essential level of simplification, the authors believe that, nowadays, the challenge becomes finding a possible way to add dexterity to such a hand without sensibly increasing its complexity. This paper proposes an evolution of the Pisa/IIT SoftHand, the SoftHand+ (Fig. 1), which moves a step in this direction. The SoftHand+, which shares much of the architecture of the original SoftHand, including its basic synergy, is actuated with two motors that are smaller w.r.t. the one used in the SoftHand. Nevertheless it can reach the same performance in terms of closure and offers one additional DOA that, thanks to the clever exploitation of friction effects, sensibly enhances its grasping and manipulation performance.

The paper is organized as follow: in section II adaptive synergies and the original Pisa/IIT SoftHand are briefly described, in section III the SoftHand+ is introduced on a theoretical level, with section IV supporting its core idea with simulative results. Finally, in section V the SoftHand+ prototype is validated in preliminary experiments.

\section{ADAPTIVE SYNERGIES \& PISA/IIT SOFTHAND}

To our extent postural synergies are substantially equivalent to the concept of basis of a vector space of the joint motions $q$, in fact it is possible to write:

$$
q=S \sigma,
$$

where $\sigma$ are the synergy variables, $q$ are the joint variables and $S$ mapping $\sigma$ into $q$, represents the synergistic basis of the joints configuration space.

\footnotetext{
${ }^{1}$ Videos available at https://goo.gl/8zYDWs
}

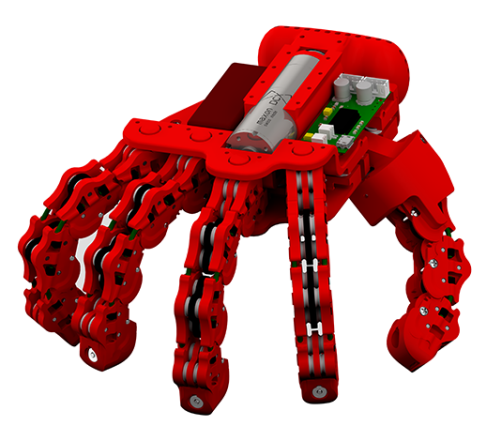

(a) Original Pisa/IIT SoftHand.

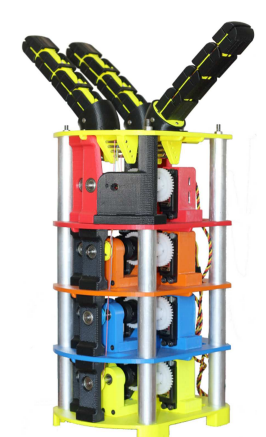

(b) THE Second Hand.
Fig. 3. Adaptive synergies based robotics hands: Pisa/IIT SoftHand integrates one DoA in a compact setup, while THE Second Hand implements four soft synergies at the cost of a cumbersome structure.

While a fully actuated synergistic space would require a full rank square matrix $S$, experimental human motor control evidence (from [14] to [15]) suggest that a reduced basis is sufficient to the hand for most of its total movement during grasping tasks. Based on this [7] proposes to generate reference motions, using a reduced set of the main postural synergies, which the real hand follows, compliantly attracted, while physically interacting with the environment. When an equilibrium is reached, the soft synergies model tells that:

$$
q=S \sigma-C J^{T} f_{e x t} .
$$

where the matrix $C$ represents the compliance and the term $J^{T} f_{\text {ext }}$ collects all the external forces acting on the hand (see also Fig. 2).

The technique of adaptive synergies, introduced in [12], is a method to derive the behavior of soft synergies from the adaptability of differential mechanisms, working on the space of self-motions to adapt to the external world. In particular assuming a hand is actuated by means of a differential mechanism with transmission distribution matrix $R$, it is possible to write

$$
R q=x
$$

where $x$ is the displacement of the motor. From kineto-static duality, we also get:

$$
\tau_{x}=R^{T} f,
$$

where, $\tau_{x}$ is the vector of torques acting on the joints and $f$ the force from the motor. Writing the equilibrium of the joint torques, which includes a linear elastic force in joint space $(-E q)$ and the contribution of external forces $\left(J^{T} f_{e x t}\right)$, one obtains:

$$
J^{T} f_{e x t}=R^{T} f-E q .
$$

Finally, combining 3 and 5 and solving yields:

$$
\begin{aligned}
q= & \left(-E^{-1}+E^{-1} R^{T}\left(R E^{-1} R^{T}\right)^{-1} R E^{-1}\right) J^{T} f_{\text {ext }}+ \\
& +E^{-1} R^{T}\left(R E^{-1} R^{T}\right)^{-1} x
\end{aligned}
$$

which can be reduced to the form of soft synergies with $x=\sigma$, by proper choice of $E$ and $R$, which can be found by 
inverting

$$
\begin{aligned}
& S=E^{-1} R^{T}\left(R E^{-1} R^{T}\right)^{-1}=R_{E^{-1}}^{+} \\
& C=E^{-1}-E^{-1} R^{T}\left(R E^{-1} R^{T}\right)^{-1} R E^{-1}=P_{R}^{\perp} E^{-1} .
\end{aligned}
$$

This method was used for the design of the Pisa/IIT SoftHand (Fig. 3(a)), where the differential mechanism is obtained using a unique tendon that actuates all joints at the same time, implementing one synergy. With the same method, it is possible to add more synergies to the hand by adding other tendons in parallel to the first one. The proof of concept of this was shown in a prototype presented in [12] (see Fig. 3(b)). The cost of doing so is undeniable in terms of dimensions and weight and derives from the necessity to replicate the routing path of the tendon, adding an independent sets of pulleys.

\section{AUGMENTATED ADAPTIVE SYNERGIES}

The model presented in the section II accounts only for elastic contributions to the force equilibrium that is, essentially, a simplification. Introducing also the effects of a generic friction contribution $\tau_{f}$, which accounts for dissipative effects, will let us augment the dexterity of the SoftHand with a lesser increment in term of mechanical complexity.

While the original SoftHand was designed by an appropriate choice of the pulley radii $(R)$ and of the elasticity of its joint $(E)$ to implement the desired synergy and minimize the impact of friction, the SoftHand+, that we introduce in this work, is designed to use friction to its own advantage.

In both the SoftHand and the SoftHand+, the main source of friction is within the pulleys that route the tendon throughout the hand. To mathematically describe the effects of friction, introduce the vectors $v$ and $T$, lying in $\mathbb{R}^{m+1}$ (where $m$ is the number of pulleys), which describe the speed and the tension of each segment of tendon between a pulley and the next one, as in Fig. 4. It is possible to calculate the value of $T_{j}$ and $v_{j}$ incrementally following the tendon route, as

$$
\left\{\begin{array}{lll}
T_{j}=T_{j-1}-V_{j}\left(v_{j}\right) & \wedge & T_{0}+T_{m}=\tau_{M} \\
v_{j}=v_{j-1}+\sum_{i=1}^{n} r_{j, i} q_{i} & \wedge & v_{0}+\dot{s}=-v_{m}-\dot{s} \\
\tau_{i}=-\sum_{j=1}^{m+1} r_{j, i} T_{j} & &
\end{array}\right.
$$

where $\tau_{M}$ is the pulling force applied by the motor on the synergy $\sigma, V_{j}\left(v_{j}\right)$ is the friction loss on the $j-t h$ pulley (depending from the $j-t h$ velocity $v_{j}$ ) and $r_{j, i}$ is equal to the radius of $j-t h$ pulley when it is on joint $i$, or to 0 otherwise.

The variable $\dot{s}$ accounts for the net slide of the tendon along its path. Please note the that $s$ is linearly independent from the joint variables $q$ (and thus also from the set of synergies implemented by $\sigma$ ) which is evident considering that constraining all the degrees of freedom of the hand, the tendon can still slide along its path, being all the pulleys idle. This does not happen in [13] because both the ends of the tendon of the original SoftHand are fixed on the same motor pulley, and thus $\dot{s} \equiv 0$. Despite the simplicity of this model, note that the number of variables tends to be large,

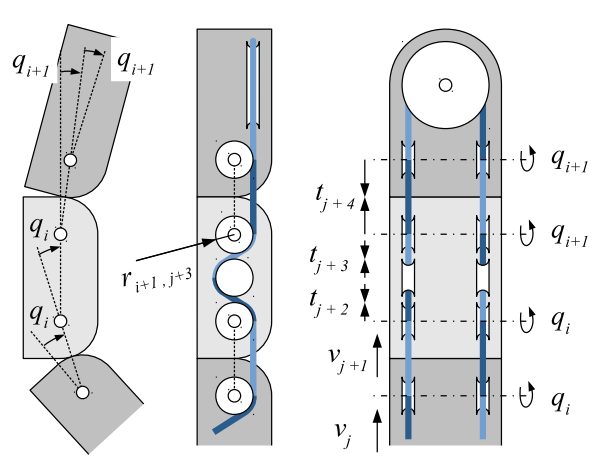

Fig. 4. Scheme of a finger of the SoftHand+ with main variables indicated. $q_{i}$ is the $i-t h$ joint angle, $v_{j}$ and $T_{j}$ are the speed and tension, respectively, of each segment of tendon along its routing, $r_{j, i}$ is the radius of the $j-t h$ pulley when it is on joint $i$, or 0 otherwise.

as an example consider that in the SoftHand, both $T$ and $v$ lie in $\mathbb{R}^{133}$.

Equation (8) can be re-written in matrix form, obtaining:

$$
\left\{\begin{array}{l}
M T+V(v)+e \tau_{M}=0 \\
M v-\Re \dot{q}=2 e \dot{s} \\
\tau=-\mathfrak{R}^{T} T
\end{array}\right.
$$

with $M \in \mathbb{R}^{m+1 \times m+1}, \mathfrak{R} \in \mathbb{R}^{n \times m+1}$ and $e=[0,0, \ldots, 0,1]^{T} \in$ $\mathbb{R}^{m+1}$. It is important to note that matrix $\mathfrak{R}$ is not the same matrix as $R$ of (3), being its $(j, i)$ element $r_{j, i}$, but has the similar role of mapping joints displacements in tendon displacements. The exact relationship between $\mathfrak{R}$ and $R$ will become clear later in the paper. Note also that $V(v)$ is a vector function which $j-t h$ element is $V_{j}\left(v_{j}\right)$.

From the previous equation it is possible to explicit the velocity distribution as

$$
v=M^{-1} \mathfrak{R} \dot{q}-e_{v} \dot{s}
$$

where $e_{v}=-2 M^{-1} e=[1,1, \ldots, 1,1]^{T}$.

Similarly, it is also possible to explicit

$$
T=-M^{-1} V(v)-M^{-1} e \tau_{M}=-M^{-1} V(v)+\frac{1}{2} e_{v} \tau_{M} .
$$

Combining (10) and (11) yields

$$
\tau=-\mathfrak{R}^{T}\left(-M^{-1} V\left(M^{-1} \mathfrak{R} \dot{q}-e_{v} \dot{s}\right)+\frac{1}{2} e_{v} \tau_{M}\right) .
$$

It is now clear how it is possible to look at $\dot{s}$ as an additional input (or DOA), with non-linear transmission distribution

$$
D(\dot{q}, \dot{s}) \triangleq-\mathfrak{R}^{T}\left(-M^{-1} V\left(M^{-1} \mathfrak{R} \dot{q}-e_{\nu} \dot{s}\right)\right) .
$$

Defining a model for the friction force $V(v)$ can help simplify the model. If, for example, we consider a dynamic Coloumblike friction model, for which

$$
V(v)=V_{\text {max }} \operatorname{sign}(v)=V_{\text {max }} \operatorname{sign}\left(M^{-1} \mathfrak{R} \dot{q}-e_{v} \dot{s}\right),
$$

(where $\operatorname{sign}(\cdot)$ is assumed component-wise), and assume the system in equilibrium (i.e. $\dot{q} \equiv 0$ ), yields the simplified expression

$$
D(\dot{s})=-\mathfrak{R}^{T}\left(M^{-1} V_{\max } \operatorname{sign}\left(e_{v} \dot{s}\right)\right)
$$


which, remembering that $e_{v}=[11 \cdots 1]^{T}$, becomes equal to

$$
D(\dot{s})=-\mathfrak{R}^{T} M^{-1} V_{\max } e_{v} \operatorname{sign}(\dot{s}) .
$$

Note that where typically friction is considered an impediment to motion, here it is exploited to generate motion, since the described effect is completely conveyed by the presence of a friction on the transmission pulleys, in other words if no friction were considered, $V(q) \equiv 0$ and the terms $D(\dot{s})$ would be null too.

Introducing the contribution of $D(\dot{s})$ in the force balance (5), we obtain:

$$
\begin{aligned}
J^{T} f_{\text {ext }} & =-\mathfrak{R}^{T} M^{-1} V_{\max } e_{v} \operatorname{sign}(\dot{s})-\mathfrak{R}^{T} \frac{1}{2} e_{v} \tau_{M}-E q= \\
& =R_{f}^{T} \operatorname{sign}(\dot{s})+R^{T} \tau_{M}-E q
\end{aligned}
$$

where, in the second row, we defined:

$$
\begin{cases}R^{T}=-\mathfrak{R}^{T} \frac{1}{2} e_{v} & \wedge u_{1}=\tau_{M} \\ R_{f}^{T}=-\mathfrak{R}^{T} M^{-1} V_{\max } e_{v} & \wedge u_{2}=\operatorname{sign}(\dot{s}) .\end{cases}
$$

Note that the first equation gives back the matrix $R$ which is the same as that of (3).

So, in analogy to section II we can define an artificial synergy-like motion associated to $\sigma_{f}$, as in:

$$
\left\{\begin{array}{l}
R q=\sigma \\
R_{f} q=\sigma_{f}
\end{array}\right.
$$

Since the expression:

$$
\left[\begin{array}{cc}
-E & {\left[R^{T} R_{f}^{T}\right.} \\
{\left[\begin{array}{c}
R \\
R_{f}
\end{array}\right]} & \emptyset
\end{array}\right]\left[\begin{array}{c}
q \\
{\left[\begin{array}{c}
u_{1} \\
u_{2}
\end{array}\right]}
\end{array}\right]=\left[\begin{array}{c}
J^{T} f_{\text {ext }} \\
{\left[\begin{array}{c}
\sigma \\
\sigma_{f}
\end{array}\right]}
\end{array}\right] .
$$

results always non singular, it leads, through block inversion, to

$$
q=\left[\begin{array}{c}
R \\
R_{f}
\end{array}\right]_{E^{-1}}^{+}\left[\begin{array}{c}
\sigma \\
\sigma_{f}
\end{array}\right]+P_{R, R f}^{\perp} E^{-1} J^{T} f_{e x t}
$$

This model explicitly derives relationships between tendon sliding and actuation units, but has the drawback of not describing the modulation of the force acting on the added synergy as a continuous phenomenon. Indeed, for the particular expression only $u_{2}= \pm 1$ it is admitted, which corresponds to:

$$
\left[\begin{array}{c}
\sigma \\
\sigma_{f}
\end{array}\right]=\left[\begin{array}{c}
R \\
R_{f}
\end{array}\right] E^{-1}\left[R^{T} R_{f}^{T}\right]\left[\begin{array}{c}
\tau_{M} \\
\pm 1
\end{array}\right]
$$

This limitation, in reality, concerns only the model used to describe the system (and, in particular, it is a consequence of assuming (14)), and does not impact the real system itself, which is subject to static friction as well. This conjecture, which is at the core of the possibility to control the SoftHand+, is supported by the simulation results that are presented in the next section.
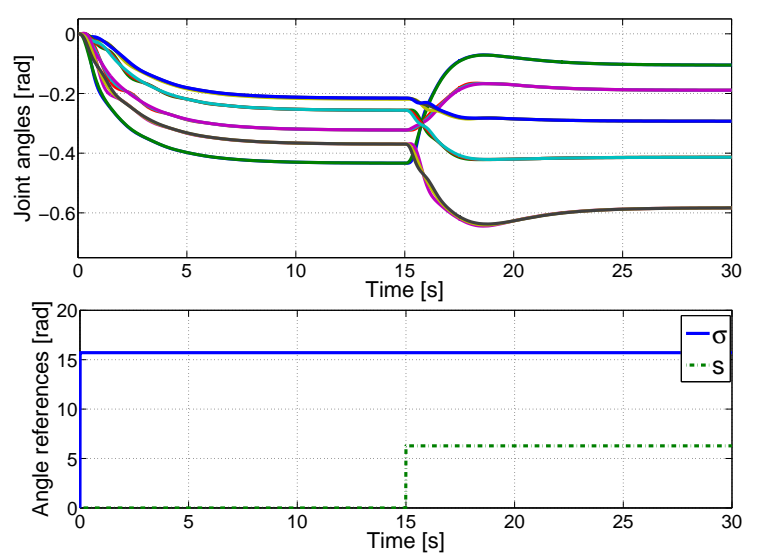

Fig. 5. Simulated joint angles evolutions on the SoftHand+. The system inputs are a step of $5 \pi \mathrm{rad}$ along the first DOA $\sigma$ at time $0 s$, then another step on the second DOA $s$, with amplitude $2 \pi \mathrm{rad}$ at time $15 \mathrm{~s}$ (abduction angles omitted for clarity, negative angles corresponds to hand closure).

\section{SIMULATION RESULTS}

Exact modeling static friction phenomena is a very complex and computationally expensive task, nevertheless, for the purpose of supporting the conjecture that (21) can be used to a broader extent than what is suggested by the constraints in (22), a simple static friction model, as that introduced in [16], is sufficient. All the simulation presented here resort to a dynamic model which includes also all the inertial and dissipation actions acting on the system and the actuator dynamics, among other effects. This fine simulation model gives easy access to the value of all the variables, in particular we can monitor the tendon tension along the hand. This makes the simulation an important tool which can be used iteratively, during the design process, to tune the shape of two motions associated with the two DOAs. A detailed description of the model used for the simulations is too long to fit in this paper and will be presented in future publications.

In Fig. 5 we present the joint angles evolution of the SoftHand+ resulting from a step input in $\sigma$ of $5 \pi \mathrm{rad}$ at $0 \mathrm{~s}$, and another step input in $s$ with amplitude $2 \pi \mathrm{rad}$ starting at $15 s$. It is clear from the figure that while the action on $\sigma$ has the same effect that it has on the Pisa/IIT SoftHand (i.e. to close the hand), the effect of $s$ corresponds to a closure on some fingers and to an opening on the others.

Fig. 6(a) presents final postures (panel a) and tensions (panel b) of simulations performed with step input in $\sigma$ of $5 \pi \mathrm{rad}$ at $0 s$, and a ramp inputs in $s$ with different slopes, starting at $5 s$. From these simulation it clearly results how the final tendon tensions, and hence postures, depend mostly on the sign of $\dot{s}$ and less on its magnitude, apparently confirming (22). Indeed the system reaches an equilibrium between potential elastic field and input action that depends mostly on dynamic coulomb friction, i.e. that accounted for by the simplified model (14).

Fig. 7, on the other hand, presents postures and tension distributions corresponding to a sequence of inputs in the 


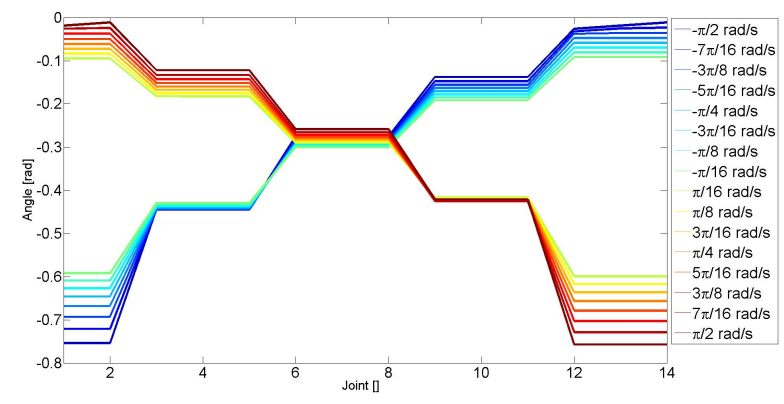

(a) Angles

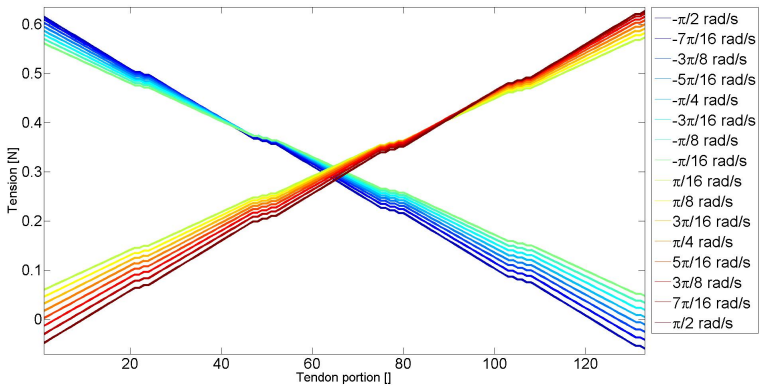

(b) Tensions

Fig. 6. Simulation results of the application of ramp inputs on the second DOA $(\dot{s}=$ const). It is possible to notice that the final hand positions and tensions get polarized among two postures, in accordance with the model described in (22) (abduction angles omitted for clarity, negative angles corresponds to hand closure).

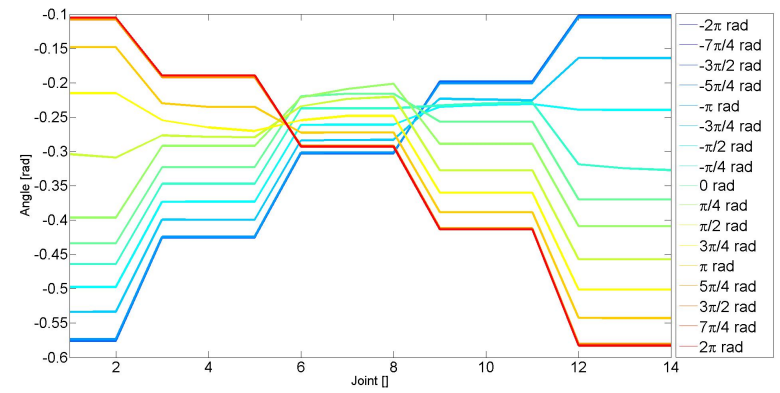

(a) Angles

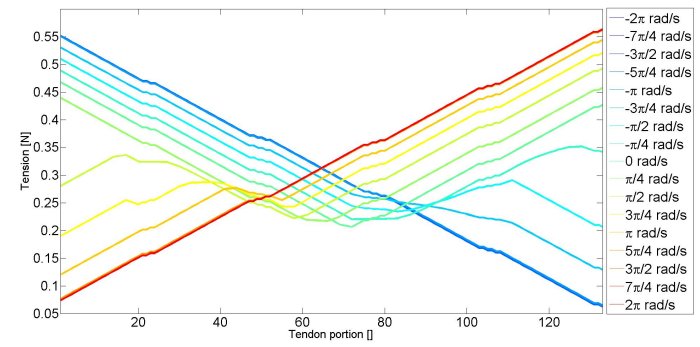

(b) Tensions

Fig. 7. Simulation results of the application of step inputs on the second DOA $(s=$ const $)$. It is possible to notice that the final hand position and tensions tend to spread between the two limit postures of figure 6 , in accordance to what conjectured at the end of section III (abduction angles omitted for clarity, negative angles corresponds to hand closure).

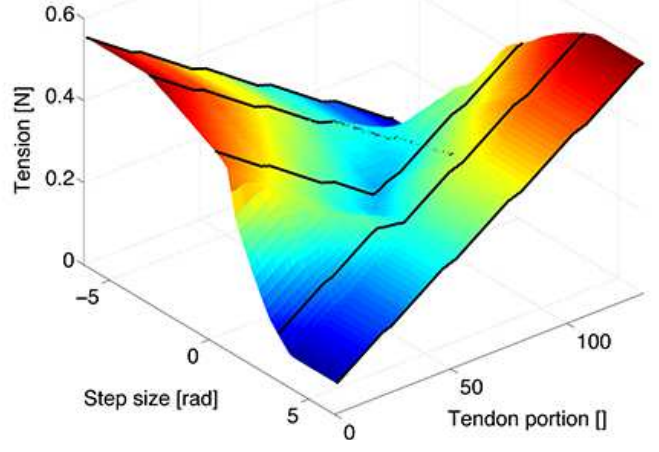

Fig. 8. Steady-state tensions distributions on the tendon with respect to changing amplitude of step input $s$. Results are obtained in 26 independent simulations. Each simulation step size differs of $\frac{\pi}{6} \mathrm{rad}$ from neighboring simulations. Tension distributions generated by step of $-2 \pi,-\pi, 0, \pi$, and $2 \pi \mathrm{rad}$ are highlighted with black lines.

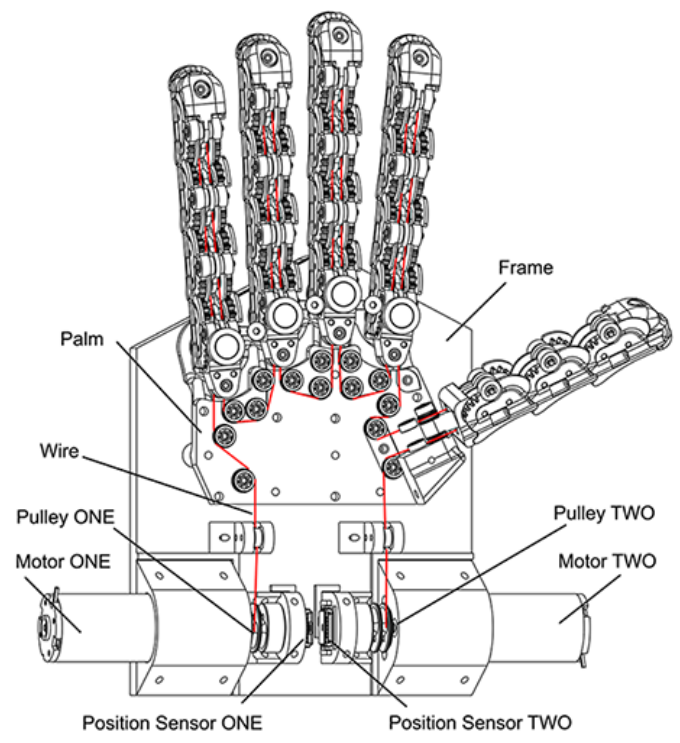

Fig. 9. Sketch of the SoftHand+ prototype components. The main subsystems are indicated by arrows and named. The red line highlights the tendon route.

form of steps of $5 \pi \mathrm{rad}$ on $\sigma$ at $0 \mathrm{sec}$ and a step on $s$ at $5 \mathrm{sec}$. These simulations demonstrate how, thanks to static friction, the entire range of postures between the two extremes of Fig. 6(a) can be achieved statically, using as control input $s$. This supports the conjecture expressed in the previous section that, indeed, control variable $s$ can be used to span with continuity over a range of positions, rather than discretely.

\section{EXPERIMENTAL RESULTS}

Following the method of augmented adaptive synergies introduced with the model of section III and validated by the simulation results of section IV, the prototype of the Pisa/IIT SoftHand+, shown in Fig. 1, was designed and built. A schematics of its main parts, highlighting its actuation and distribution mechanism is shown in Fig. 9. The prototype 


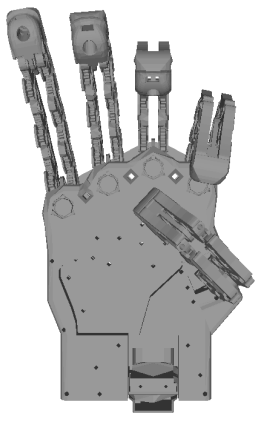

(a) Model, $s=\pi \mathrm{rad}$

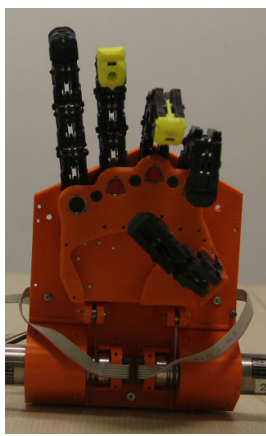

(c) Real, $s=\pi \mathrm{rad}$

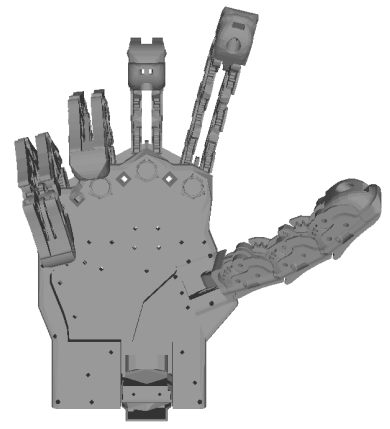

(b) Model, $s=-\pi \mathrm{rad}$

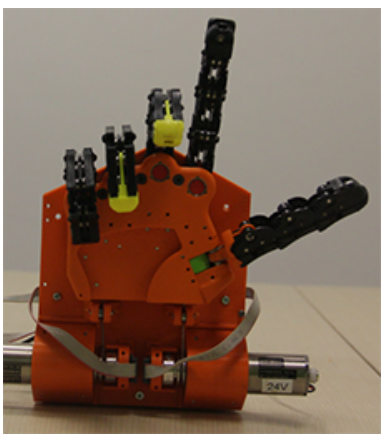

(d) Real, $s=-\pi \mathrm{rad}$

Fig. 10. Postures of the Pisa/IIT SoftHand+, model versus experimental. The posture correspond to a constant value of $\sigma=\pi \mathrm{rad}$ and two different values of $s= \pm \pi \mathrm{rad}$. Postures forecast by the model in simulation (top row) are compared with experimental results (bottom row).

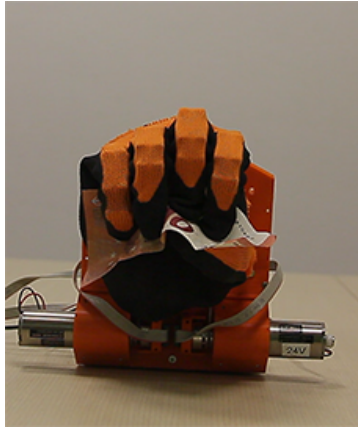

(a) One DOA grasp: banknote
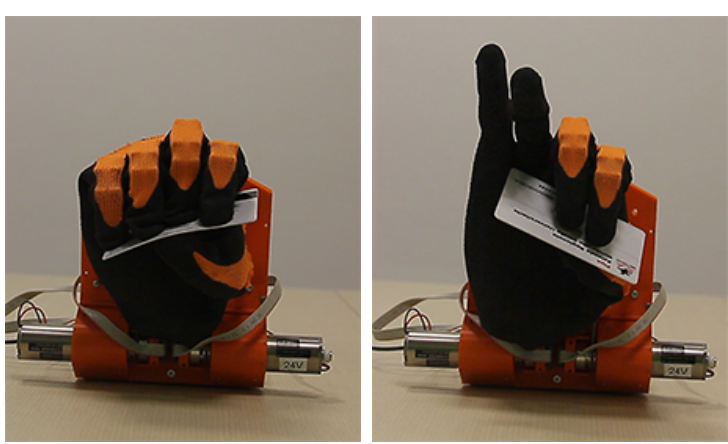

(c) One DOA grasp: credit card(d) Two DOAs grasp: credit card

Fig. 11. Grasping capabilities comparison between one DOA and two DOA. The two grasps on the left side of the figure are obtained using the first DOA of the SoftHand+, which corresponds to the first adaptive synergy, i.e. the same implemented by the SoftHand. The second DOA enables more natural grasps, in particular when grasping small and/or flat objects, as with the bank note and the credit card of these examples. uses two MAXON DC-X 22s $24 \mathrm{~V}$ motors mounting 86 : 1 gearboxes, characterized by $15 \mathrm{~W}$ of continuous output power. The position of the output shafts (after the gearboxes) is acquired using two digital magnetic sensors from Austrian Microsystems. The motors are controlled with a custom electronic board derived from the design of the control board of the qbmove actuators, whose open-source schematics is available online [17]. Note that the two DOAs of the SoftHand+ correspond to the sum and the difference of the position of the two motors.

The introduction of the proposed actuation mechanism equips the hand with various novel skills, such as the possibility to obtain new grasp postures, to redistribute grasp force and some basic in-hand manipulation skills. Fig. 10 presents the posture resulting from the sequence of a step on $\sigma$ and a step on $s$ of $2 \pi$ and $-2 \pi$. The resulting behavior is coherent with the one coming from the simulations, shown on the same figure, on top.

It is worthwhile to notice the similarity of the movement associated with $s$ with the second grasp synergy presented in [18] and with the third in [14]. Unfortunately, at the moment, it is not possible to assess a formal comparison of the three movements, given some slight differences in the boundary condition of the experiments, and in particular of the hands kinematics. We demand to future studies for such a comparison.

In Fig. 11 the SoftHand+ exhibits grasping postures that allow it to obtain easier and more natural grasps of small objects (when compared with the original SoftHand). Another possibility of the SoftHand+ is that of using the second actuation input to generate different force distributions on already grasped objects. Fig. 12 shows this effect, with the SoftHand+ deforming a pre-grasped sponge. Finally the basic SoftHand+ manipulation skills are shown in Fig. 13 to rotate a test disc, and are applied in Fig. 14 to pour some coffee from a cup using in-hand manipulation only.

Finally, we point the reader to the additional demonstration material presented in the article video footage.

\section{CONCLUSIONS}

This paper proposed an evolution of the adaptive synergies actuation system that powers the Pisa/IIT SoftHand. Such Evolution, based on friction exploitation, let us design a novel prototype of underactuated robot hand with two DOAs, named Pisa/IIT SoftHand+. A simplified mathematical model was introduced to justify the new actuation system and simulation results were presented alongside to it, to support the intuition behind the new design. A first prototype was presented and briefly validated, based on grasping and manipulation examples of some objects. Future work will consist in developing a more stable version of SoftHand+, and in studying the similarities between the newly implemented degree of actuation and human synergies.

\section{ACKNOWLEDGMENT}

The authors want to thank Andrea Di Basco, Lorenzo Malagia and Fabio Bonomo for their really valuable support 

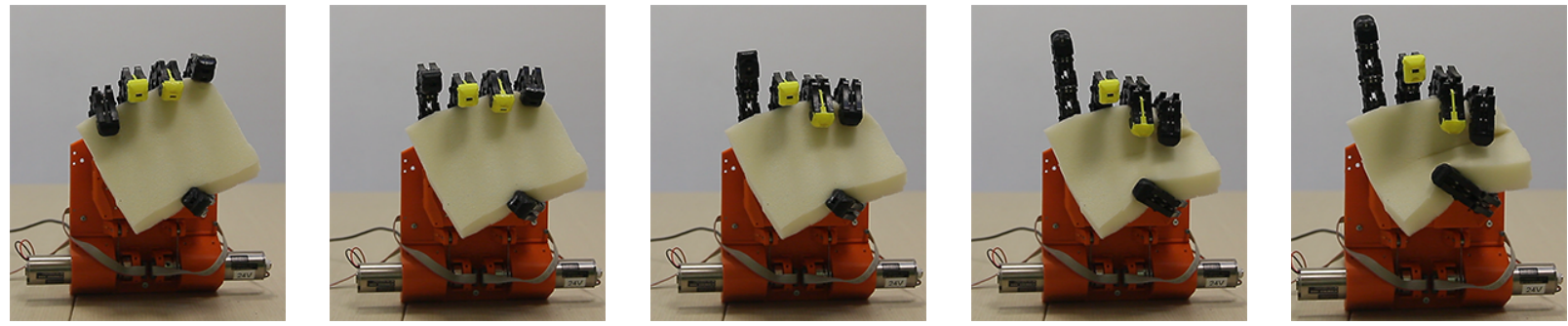

Fig. 12. Post-grasp force re-distribution with the second DOA demonstrated on a sponge, photo-sequence (from left to right).
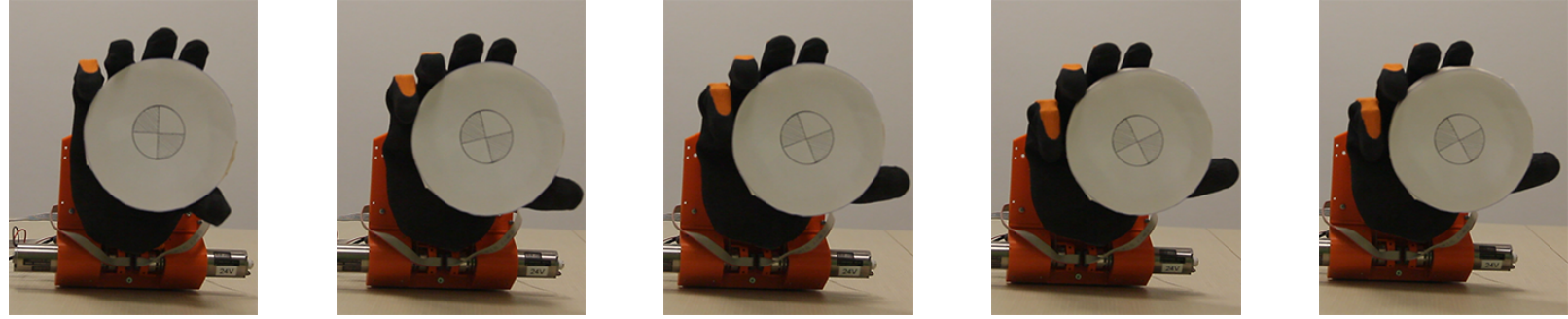

Fig. 13. In-hand manipulation: rotation of a disk, photo-sequence (from left to right).
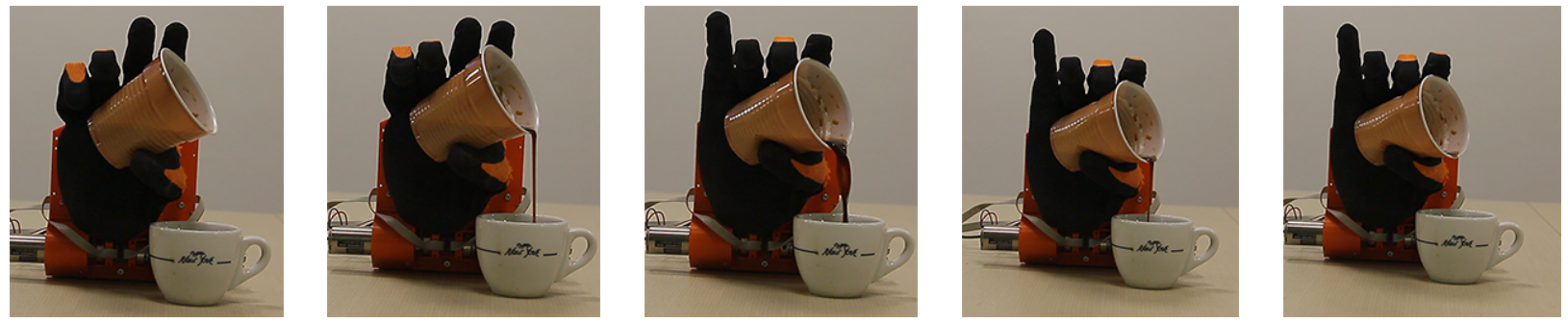

Fig. 14. In-hand manipulation: pouring coffee from a cup, photo-sequence (from left to right).

in the development of the hardware prototype. The authors wish to give credit to Hamal Marino for its help in figure realization.

\section{REFERENCES}

[1] M. Grebenstein, A. Albu-Schäffer, T. Bahls, M. Chalon, O. Eiberger, W. Friedl, R. Gruber, S. Haddadin, U. Hagn, R. Haslinger, et al., "The dlr hand arm system," in Robotics and Automation (ICRA), 2011 IEEE International Conference on, pp. 3175-3182, IEEE, 2011.

[2] L. Birglen, C. M. Gosselin, and T. Laliberté, Underactuated robotic hands, vol. 40. Springer Science \& Business Media, 2008.

[3] T. Laliberté, L. Birglen, and C. Gosselin, "Underactuation in robotic grasping hands," Machine Intelligence \& Robotic Control, vol. 4, no. 3, pp. 1-11, 2002.

[4] S. Hirose, "Connected differential mechanism and its applications," Proc. 2nd ICAR, pp. 319-326, 1985.

[5] N. A. Bernstein, The Co-ordination and regulation of movements. Pergamon Press Ltd., first english edition ed., 1967.

[6] M. L. Latash, Fundamentals of motor control. Academic Press, 2012.

[7] A. Bicchi, M. Gabiccini, and M. Santello, "Modelling natural and artificial hands with synergies," Philosophical Transactions of the Royal Society B: Biological Sciences, vol. 366, no. 1581, pp. 31533161, 2011.

[8] C. Y. Brown and H. H. Asada, "Inter-finger coordination and postural synergies in robot hands via mechanical implementation of principal components analysis," in Intelligent Robots and Systems, 2007. IROS 2007. IEEE/RSJ International Conference on, pp. 2877-2882, IEEE, 2007.

[9] M. Ciocarlie, C. Goldfeder, and P. Allen, "Dexterous grasping via eigengrasps: A low-dimensional approach to a high-complexity problem," in Robotics: Science and Systems Manipulation WorkshopSensing and Adapting to the Real World, Citeseer, 2007.
[10] T. Wimboeck, C. Ott, and G. Hirzinger, "Passivity-based object-level impedance control for a multifingered hand," in Intelligent Robots and Systems, 2006 IEEE/RSJ International Conference on, pp. 4621-4627, IEEE, 2006.

[11] K. Xu, H. Liu, Y. Du, and X. Zhu, "Design of an underactuated anthropomorphic hand with mechanically implemented postural synergies," Advanced Robotics, vol. 28, no. 21, pp. 1459-1474, 2014.

[12] G. Grioli, M. Catalano, E. Silvestro, S. Tono, and A. Bicchi, "Adaptive synergies: an approach to the design of under-actuated robotic hands," in Intelligent Robots and Systems (IROS), 2012 IEEE/RSJ International Conference on, pp. 1251-1256, IEEE, 2012.

[13] M. G. Catalano, G. Grioli, E. Farnioli, A. Serio, C. Piazza, and A. Bicchi, "Adaptive synergies for the design and control of the pisa/iit softhand," The International Journal of Robotics Research, vol. 33, no. 5, pp. 768-782, 2014.

[14] M. Santello, M. Flanders, and J. F. Soechting, "Postural hand synergies for tool use," The Journal of Neuroscience, vol. 18, no. 23, pp. 1010510115, 1998.

[15] M. Gabiccini, A. Bicchi, D. Prattichizzo, and M. Malvezzi, "On the role of hand synergies in the optimal choice of grasping forces," Autonomous Robots, vol. 31, no. 2-3, pp. 235-252, 2011.

[16] V. Hayward and B. Armstrong, "A new computational model of friction applied to haptic rendering," in Experimental Robotics VI, pp. 403-412, Springer, 2000.

[17] "Natural machine motion initiative." http://www. naturalmachinemotioninitiative.com/. website visited on July 2015.

[18] M. Gabiccini, G. Stillfried, H. Marino, and M. Bianchi, "A datadriven kinematic model of the human hand with soft-tissue artifact compensation mechanism for grasp synergy analysis," in Intelligent Robots and Systems (IROS), 2013 IEEE/RSJ International Conference on, pp. 3738-3745, IEEE, 2013. 